The Galvanometer.-For use with multiple thermoelements of 20 to $5^{\circ}$ couples a galvanometer of the following specifications ${ }^{1}$ is recommended: resistance $30 \mathrm{ohms,} \mathrm{external} \mathrm{critical} \mathrm{damping} \mathrm{resistance} 400$ ohms, period $7 \mathrm{sec}$, sensitivity $4 \mathrm{~mm}$. per microvolt with the damping resistance in series. The potentiometer is usually used with several thermocouples which have different resistances. Instead of changing $\mathrm{Z}$ (Fig. I) to correspond to the resistance of each thermocouple, it is fixed at 400 (C. D. R. of galvanometer)-105.5 (resistance of remainder of external galvanometer circuit) and an auxiliary resistance, $Q$, placed in series with each thermocouple. This resistance $Q$ is wound of copper wire so as to be free of thermoelectric forces, and has such a value that its resistance added to that of the thermocouple equals $Z$.

For the above galvanometer the resistance $S$ should have the value 400 ohms, and $\mathrm{H} 3572 \mathrm{ohms}^{2}$ in order that the galvanometer can be set to give $1 / 10$ sensitivity.

Our instrument as finally set up has no detectable thermoelectric parasitics, and only an extremely constant leakage e. m. f. of slightly less than o.I microvolt.

Barxelefy, Cal.

[CONTribution from the School of Chemistry, University of PtTtsburgh, PITTSBURgh, PA.]

\title{
THE TEMPERATURE EFFECT IN DIALYSIS AND A SIMPLE RAPID DIALYZER. ${ }^{3}$
}

By Marks NeIdLE.

Received April 27, 1916.

The speed of dialysis depends upon the following factors: First, the nature of the membrane; second, the area of the membrane which is in contact with liquid on both sides; third, the difference in concentration of diffusible substances in internal and external liquids close to the membrane; fourth, the temperatures of the internal and external liquids.

By utilizing, especially the third and fourth principles, it is possible to prepare large quantities of inorganic hydrosols, containing only minute amounts of electrolytes, in a comparatively short time. The method consists in suspending a parchment paper membrane of about one liter capacity in a two liter beaker containing about a liter of the solution to be dialyzed. Distilled water is run at a fairly constant rate into the membrane, which is maintained a little more than half full by means of an automatic syphon. The colloidal solution which is in the beaker is heated to any desired temperature $\left(70-90^{\circ}\right)$.

1 For use with low-resistance couples the critical damping resistance can be reduced to 150 ohms with an increase of sensitivity.

${ }^{2}$ For a galvanometer 150 ohms C. D. R., $S=150$ ohms, and $H=1625$ ohms.

${ }^{3}$ Presented at the spring meeting of the American Chemical Society, 1916. 
Wobbe $^{1}$ and Nicolardot ${ }^{2}$ speak of a process of hot dialysis but they neither describe their methods nor discuss the advantages of dialyzing in this way. In connection with a device, which is essentially a modified Soxhlet apparatus, for the quantitative and rapid dialysis of small volumes of colloidal solutions, Golodetz ${ }^{3}$ mentions that the dialysis membrane which is above the boiling flask is heated to about $40^{\circ}$ to $50^{\circ}$, this being favorable to rapid dialysis.

Beyond these mere statements it seems that the temperature effect in dialysis has been completely neglected. Its importance is evident from the fact that the simple apparatus represented in the diagram gives in twenty days a hydrous ferric oxide hydrosol, as pure as, if not purer, than the same hydrosol prepared by dialysis in the cold for six months. A colloidal solution satisfactory for most purposes may be obtained in eight to ten days, while the usual method requires about a month.

The temperature effect becomes apparent as soon as we regard the colloidal solution as a two-phase system in. which the electrolyte is distributed between the disperse phase and the dispersion medium according to the laws of adsorption. If the dialyzing membrane is impermeable to the disperse phase, only the

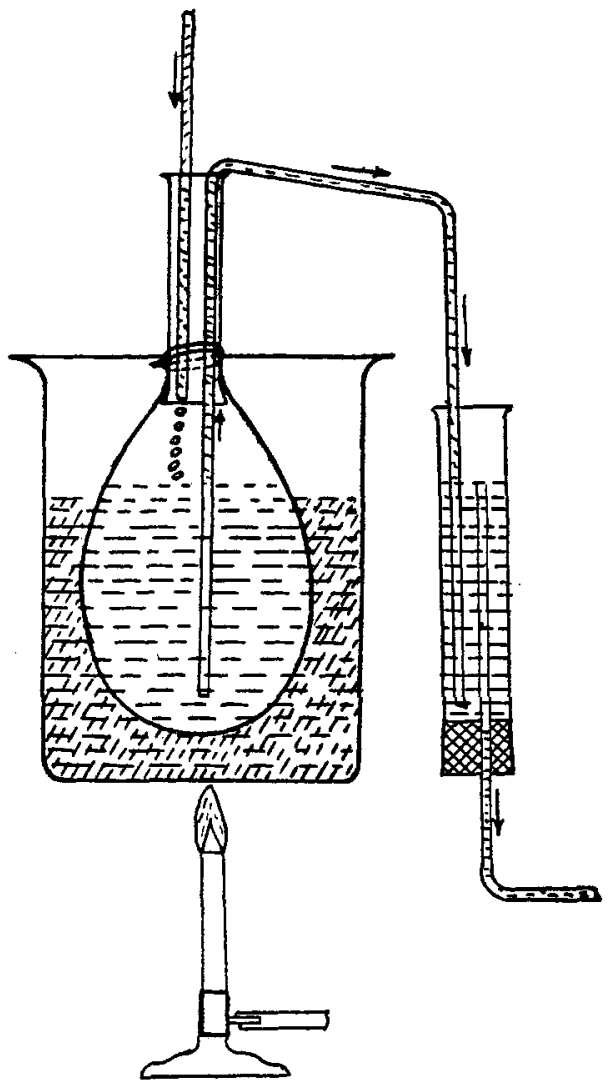
electrolyte which is not adsorbed can diffuse directly. Now, if the colloida! solution is heated, this portion of the electrolyte will diffuse more rapidly through the membrane and since the diffusion coefficient ${ }^{4}$ increases about $2 \%$ per degree, the effect of a temperature increase of $50-70^{\circ}$ will be very considerable.

As the electrolyte in the dispersion medium diffuses through the mem-

${ }_{1}^{1}$ Pharm. Centr., H., 40, 793 (1899).

${ }^{2}$ Ann. chim. phys., 366, 348 (1905).

${ }^{3}$ Z. physiol. Chem., 86, 315-24 (1913).

4 Nernst, Theoretische Chemie, 7 th ed., p. 400. 
brane, the equilibrium of distribution of the electrolyte is disturbed, and part of the adsorbed electrolyte diffuses into the dispersion medium from which it can now dialyze. Heating accelerates the re-establishment of equilibrium and consequently hastens dialysis.

Furthermore, in general, adsorption decreases ${ }^{1}$ considerably with rise in temperature. For a given total concentration of electrolyte in a colloidal solution at equilibrium there will be more of it in the dispersion medium if the solution is heated, and since diffusion through the membrane depends only on the concentration of the electrolyte in the dispersion medium, heating should be favorable to rapid dialysis.

By placing the solution to be dialyzed in the outside vessel (the beaker) and heating, the large increase in volume due to osmosis, which results in the usual procedure, is avoided. An equilibrium sets in between evaporation from the colloidal solution and osmosis of water into it through the membrane, and the volume remains practically constant. Or, if desired, the solution may be concentrated during dialysis.

The steady flow of distilled water inside the dialyzer helps dialysis by maintaining a maximum difference of concentration of diffusible substances on both sides of the membrane, and also by maintaining within the latter a lower temperature than outside, which introduces the Soret ${ }^{2}$ phenothenon.

Our experience is that parchment paper membranes of about one liter capacity are far easier to prepare than collodion membranes of the same size. They stand rough usage, a temperature of over $90^{\circ}$ for a long period, and cost very little.

Pittsaurgh, Pa.

\section{ON THE EQUILIBRIA OF MERCURIC CHLORIDE WITH OTHER CHLORIDES. \\ By G. A. Linfart. \\ Received March 6, 1916.}

In a recent paper ${ }^{3}$ it is shown that the association of mercuric chloride in water solution depends both on the concentration and the temperature, and that the degree of association ranges, in saturated solutions, from $6 \%$ at $0^{\circ}$ to $58 \%$ at $100^{\circ}$. It is, therefore, not surprising that in the absence of these data, previous investigators were not able to account for the abnormal behavior of mercuric chloride in water solution with reference to its molecular weight, distribution ratio, and solubility. Of particular interest are the attempts made by several investigators to establish the type or types of complex compounds which mercuric chloride

1 Freundlich Kapillarchemie, 169-173.

${ }^{2}$ Nernst, Theoretische Chemie, 7 th ed., p. 80r.

3 This Journal, 37, $25^{8}$ (IgI5). 